\title{
Etude de l'influence préalable de différentes enzymes amylolytiques sur la teneur en résidu NDF d'aliments du bétail
}

\author{
Sylvie GIGER, P. THIVEND *, D. SAUVANT, \\ Michelle DORLEANS, Paulette JOURNAIX * \\ INRA, Station de Nutrition et Alimentation \\ INA Paris-Grignon, Centre de Recherches de Paris-Grignon \\ 16, rue Claude-Bernard, F 75231 Paris Cedex 05 \\ * INRA, Laboratoire de la Digestion des Ruminants \\ Centre de Recherches de Clermont-Ferrand \\ Theix, F 63122 Ceyrat
}

\begin{abstract}
Résumé
La méthode initiale de Van Soest (VAN SoEst \& WinE, 1967) conduit à une surestimation de la teneur en constituants pariétaux (évaluée par la teneur en résidu NDF), notamment dans le cas des aliments riches en amidon. Il est possible de pallier cette carence en utilisant une $\alpha$ amylase d'origine bactérienne.

L'objet de la présente étude consiste à comparer l'action de trois enzymes amylasiques, utilisées à des concentrations supérieures à celles généralement employées, sur la teneur en résidu NDF de l'échantillon, sur le degré de contamination du résidu NDF par l'amidon, ainsi que sur les éventuelles actions qui ne seraient pas d'ordre amylasique de ces enzymes.

L'amylase A 1278 de chez Sigma, employée à une température de $45^{\circ} \mathrm{C}$, permet d'extraire pratiquement tout l'amidon des différents échantillons, sauf dans le cas des graines de légumineuses. Elle fournit des résultats satisfaisants et équivalents à ceux issus de l'utilisation de la A 6505, mais possède des activités cellulasique et hémicellulasique. Cette étude a permis l'élaboration d'un protocole de travail dans le cas des aliments simples ou composés, utilisés en alimentation animale, qui permet de résoudre les difficultés éventuelles de filtration liées à la teneur en amidon.
\end{abstract}

Mots clés: Paroi végétale, estimation, prétraitement amylasique, contaminations glucidiques.

\section{Introduction}

La méthode d'estimation des constituants pariétaux par pesée du résidu NDF, obtenu après l'action d'un détergent neutre (VAN SOEST \& WINE, 1967), a été conçue pour des fourrages. Or, les détergents utilisés ne permettent pas de solubiliser complètement l'amidon quand celui-ci est présent en grande quantité dans l'échantillon, ce qui est le cas de certains aliments concentrés. Il en résulte d'une part, un allongement important de la durée de filtration après l'attaque par la solution au détergent neutre (Giger et al., 1979), et, d'autre part, une estimation par excès de ces constituants pariétaux. 
Afin de pallier cette carence, RoberTSON \& VAN Soest (1977) ont proposé l'utilisation d'une amylase bactérienne extraite de Bacillus subtilis $(1.4 \alpha \mathrm{D}$-glucane, 4glucanohydrolase E.C. $N^{*} 3.2 .1 .1$., A 6505 commercialisée par Sigma). Cette enzyme n'étant plus disponible, il s'est avéré nécessaire de chercher une enzyme de remplacement. L'objet de la présente étude est donc d'étudier l'action de trois enzymes amylolytiques utilisées dans différentes conditions, sur la teneur en résidu NDF d'échantillons d'aliments destinés aux animaux domestiques, sur le degré de contamination du résidu NDF par l'amidon ainsi que sur les éventuelles actions, qui ne seraient pas d'ordre amylasique, de ces enzymes.

\section{Matériel et méthodes}

\section{A. Choix des échantillons}

Cette étude porte sur onze échantillons choisis de manière à couvrir une large gamme de teneurs en amidon (tabl. 1) et en constituants pariétaux, de telle sorte qu'ils soient aussi représentatifs que possible des principaux types de matières premières utilisées en alimentation animale. Ce sont des céréales ou leurs sous-produits (maïs, blé, orge, escourgeon, avoine, son, drèches de brasserie), des protéagineux (pois, féverole), ainsi qu'un aliment dépourvu d'amidon (pulpe de betteraves) et un aliment composé (type concentré vache laitière).

\section{TABleau 1}

Teneurs en amidon des échantillons et résidus $N D F$.

Starch content of samples and NDF residues.

\begin{tabular}{|c|c|c|c|c|c|c|}
\hline \multirow{3}{*}{$\begin{array}{l}\text { Echantillon } \\
\text { Sample }\end{array}$} & \multirow{3}{*}{$\begin{array}{l}\text { Dans } \\
\text { l'aliment } \\
\% \text { of } \\
\text { initial } \\
\text { sample }\end{array}$} & \multicolumn{5}{|c|}{$\begin{array}{l}\text { Dans le résidu NDF } \\
\% \text { of } N D F \text { residue }\end{array}$} \\
\hline & & \multirow{2}{*}{$\begin{array}{l}\text { Témoin } \\
\text { Standard }\end{array}$} & \multirow{2}{*}{ A 6505} & \multicolumn{2}{|c|}{ A 1278} & \multirow{2}{*}{$\begin{array}{c}\text { Calbio- } \\
\text { chem }\end{array}$} \\
\hline & & & & T 90 & T 45 & \\
\hline Maïs - Maize & 71,7 & 18,4 & 3,7 & 5,3 & 5,5 & 9,8 \\
\hline Blé - Wheat & 65,7 & 19,2 & 1,8 & 14,0 & 7,1 & 8,9 \\
\hline Orge - Barley & 59,1 & 18,4 & 2,5 & 2,1 & 1,7 & 9,6 \\
\hline Escourgeon - Winter Barley .. & 57,9 & 26,1 & 1,9 & 2,7 & 2,3 & 13.0 \\
\hline Avoine - Oats $\ldots \ldots \ldots$ & 53,3 & 5,1 & 1,0 & 1,0 & 0,8 & 1,6 \\
\hline Pois - Peas ... & 44,1 & 50,4 & 40,7 & 36,5 & 37,0 & 39,1 \\
\hline $\begin{array}{l}\text { Aliment composé } \ldots \ldots \ldots \\
\text { Compound Feed }\end{array}$ & 39,5 & 28,7 & 1,0 & 2,4 & 1,8 & 0,2 \\
\hline Féverole - Field beans . . . . & 37,5 & 77,8 & 15,6 & 14,9 & 25,2 & 24,0 \\
\hline Son - Bran $\ldots \ldots \ldots \ldots$ & 16,8 & 4,0 & 1,3 & 3,2 & 1,5 & 3,1 \\
\hline $\begin{array}{l}\text { Drèches de brasserie } \ldots \ldots \\
\text { Brewers grain }\end{array}$ & 3,3 & 1,8 & 1,0 & 1,7 & 1,9 & 0,9 \\
\hline $\begin{array}{l}\text { Pulpes de betteraves } \ldots \ldots \\
\text { Sugar beet pulp }\end{array}$ & 1,1 & 1,3 & 0,8 & 1,1 & 1,0 & 2,3 \\
\hline
\end{tabular}

Tous les résultats sont exprimés en \%/MS - All results are expressed as \%/DM. 


\section{B. Nature des enzymes testées et justification de leur choix}

Les trois enzymes testées sont des $\alpha$-amylases extraites de la souche bactérienne Bacillus subtilis. L'une est commercialisée par Calbiochem ${ }^{(*)}$, et les deux autres (A 6505 et A 1278) par Sigma ${ }^{(* *)}$. L'enzyme A 6505, proposée par RoberTson \& VAN Soest (1977), est celle qui a été utilisée dans les premières études sur les aliments concentrés (Giger, 1979) et est souvent considérée comme enzyme de référence (Mascarenhas Ferreira, Kerstens \& Gast, 1983).

L'enzyme A 1278 est présentée par la firme Sigma comme étant la remplaçante de l'enzyme A 6505 et devant être utilisée dans les mêmes conditions. Le choix de la troisième enzyme, celle de Calbiochem, réside dans son degré de purification, a priori supérieur à celui des autres enzymes testées.

\section{Mode d'obtention du résidu NDF}

Dans la méthode initiale (VAN SoEsT \& WinE, 1967) le résidu NDF est obtenu par la solubilisation à ébullition d'un échantillon de 1 gramme dans une solution détergente à pH neutre (NDS). La modification apportée par RoBerTSON \& VAN SOEST (1977) consiste à introduire l'enzyme amylasique après la première demi-heure d'hydrolyse dans la solution NDS, à raison de $40 \mathrm{mg} /$ échantillon. Avec ce procédé, l'enzyme est inactivée rapidement dans la solution au détergent neutre remise à ébullition, du fait de la présence d'agents chimiques comme l'EDTA (Mascarenhas Ferreira, Kerstens \& GAST, 1983) et de l'augmentation rapide de la température jusqu'à une valeur voisine de $100^{\circ} \mathrm{C}$. De plus, la quantité d'enzyme était insuffisante. Ceci nous a conduit à proposer de faire une première hydrolyse à l'eau bouillante pendant 10 minutes, pour empeser l'amidon et préparer ainsi l'échantillon à l'attaque par l'amylase, à filtrer au maximum le surnageant, à compléter avec de l'eau distillée jusqu'à $100 \mathrm{ml}$ dans lesquels on ajoute $250 \mathrm{mg}$ d'enzyme par échantillon qu'on laisse agir pendant une demi-heure à 80 "C (Giger, 1980). Après élimination du surnageant par filtration, l'échantillon subit l'attaque par la solution NDS, telle qu'elle est effectuée pour les fourrages.

Une étude préliminaire a mis en évidence que l'enzyme A 1278 était moins active que la A 6505, lorsqu'elle est utilisée dans les mêmes conditions. De ce fait, nous avons testé cette enzyme aux températures de $80^{\circ} \mathrm{C}$, qui est la température habituelle d'utilisation de la A 6505 et celle préconisée par Sigma (communication personnelle), et de $45^{\circ} \mathrm{C}$, qui est proche des températures d'action optimale enzymatique et, de plus, d'obtention facile dans le cadre d'un protocole d'analyse de routine. Nous avons également essayé d'extraire l'enzyme de son support par traitement préalable de l'enzyme par une solution tampon d'acétate de sodium-acide acétique $0,05 \mathrm{M}, \mathrm{pH} 4,7$ à $250 \mathrm{mg} / \mathrm{l}$ de tampon (C.E.E., 1981). La quantité d'enzyme utilisée est de $250 \mathrm{mg} /$ échantillon.

L'enzyme de chez Calbiochem a été utilisée avec le même mode opératoire que la A 6505 , à raison de $16,8 \mathrm{mg}$ d'enzyme par échantillon. 


\section{Dosage de l'amidon}

L'amidon a été dosé sur les aliments et sur les résidus NDF par la méthode de Thivend, Mercier \& Guilbot (1965). Le principe de cette méthode consiste à transformer quantitativement et spécifiquement l'amidon de l'échantillon en glucose par action d'une $\alpha$ 1-6 glucosidase, puis de doser le glucose ainsi formé par une méthode enzymatique utilisant une glucose-oxydase.

\section{Résultats et discussion}

\section{A. Influence des différents traitements sur la solubilisation de l'amidon}

La teneur en amidon du résidu NDF a été mesurée pour les cinq traitements suivants appliqués aux échantillons :

- pas de traitement enzymatique préalable à l'attaque par la NDS ;

- traitement par l'enzyme A 6505 ;

- traitement par l'enzyme A 1278, non extraite, aux températures de 45 et $80^{\circ} \mathrm{C}$;

- traitement par l'enzyme de Calbiochem.

Les résultats obtenus sont récapitulés dans le tableau 1. En l'absence de traitement enzymatique, l'amidon peut représenter de 1 p. 100 (pulpe de betteraves ou drèches de brasserie) à plus des trois quarts (féverole) du résidu pariétal. Les graines de légumineuses (pois et féverole) sont moins sensibles à l'action des amylases utilisées que les autres échantillons. Ceci est vraisemblablement lié à une différence dans la structure de l'amidon, qui varie en fonction des familles végétales (DuPRAT et al., 1980).

Il n'y a pas de relation entre le pourcentage d'amidon initial (exprimé en p. 100/ MS) et le pourcentage d'amidon non éliminé par le réactif au détergent neutre (tabl. 2). Cependant, la part de l'amidon initial qui se trouve dans le résidu NDF est faible (inférieur à 2 p. 100 de l'amidon initial) pour les prétraitements avec les enzymes A 6505 et A 1278, si on excepte le cas des légumineuses (6 p. 100).

\section{B. Influence des différents traitements sur les teneurs en résidu NDF}

Les différents traitements enzymatiques ont pour effet de diminuer significativement la teneur en résidu NDF (tabl. 2). Ainsi, la diminution par rapport au traitement au détergent neutre seul est respectivement de :

- 13,3 p. 100/MS (A 6505);

- 11,6 p. 100/MS (A 1278 sans extraction et à température élevée) ;

- 12,9 p. 100/MS (A 1278 sans extraction et à température basse) ;

- 9,9 p. 100/MS (A 1278 avec extraction et à température élevée) ;

- 12,0 p. 100/MS (A 1278 avec extraction et à température basse) ;

- 10,1 p. 100/MS (Calbiochem). 


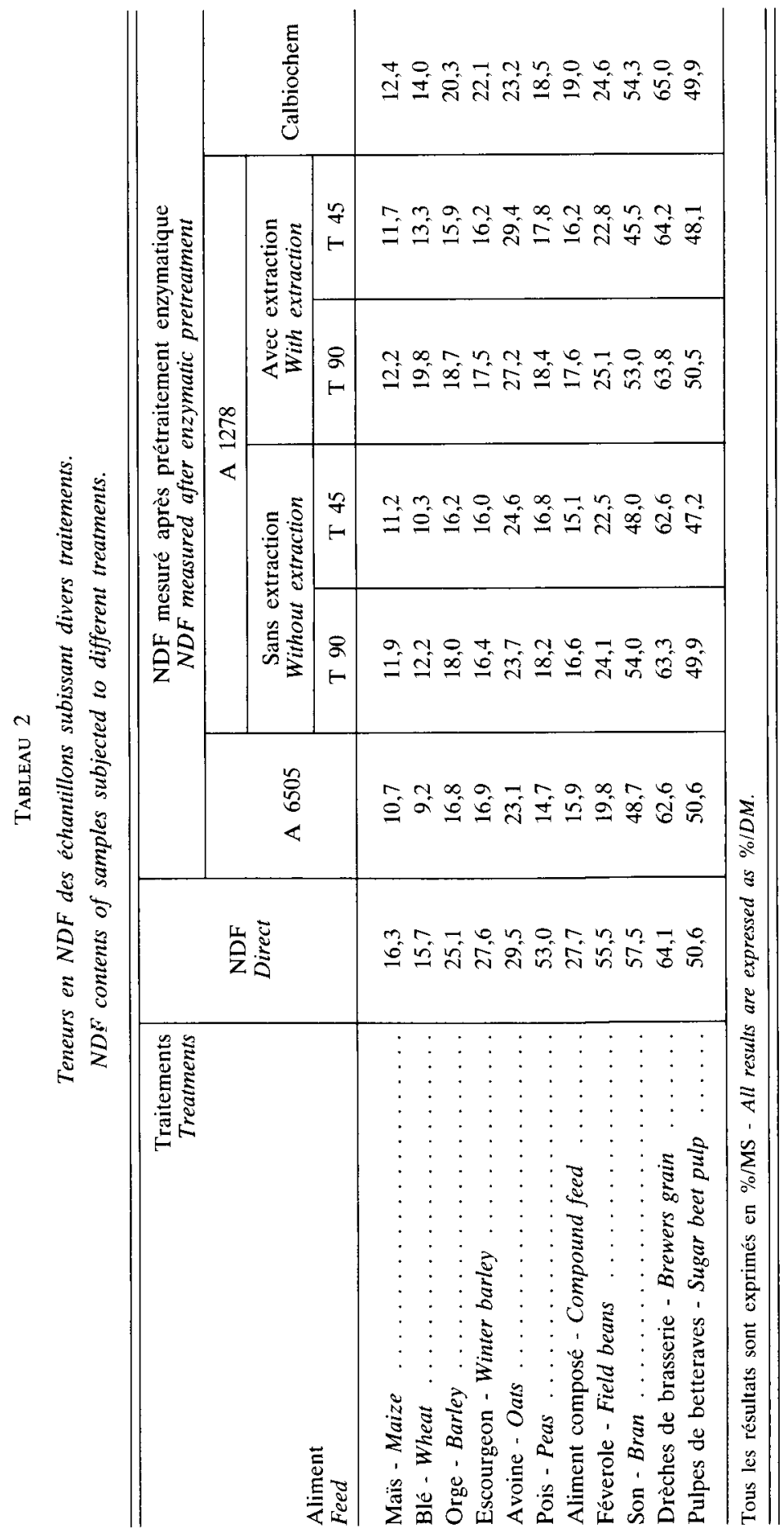


Une analyse de variance sur l'ensemble des résultats concernant les échantillons qui ont subi un traitement par une amylase montre que cette diminution est significativement influencée par :

- la nature du traitement enzymatique : l'enzyme la plus active est la A 6505, suivie par la A 1278 utilisée sous forme brute et à température basse ;

- la nature de laliment : cet effet est lié au protocole expérimental qui incluait dans l'étude des aliments de teneurs en résidus NDF très variées ;

- par ailleurs, une interaction enzyme-aliment, statistiquement significative, a été mise en évidence (tabl. 2). Les aliments qui présentent l'interaction la plus marquée sont le blé, l'avoine et le son, ce qui signifie qu'ils réagissent différemment des autres aliments aux traitements subis. L'enzyme A 1278 , après extraction et à température de $45^{\circ} \mathrm{C}$ a une activité variable suivant les aliments.

Une étude particulière a été effectuée pour l'enzyme A 1278 qui a été utilisée selon quatre protocoles différents. Cette enzyme agit significativement plus à la température de $45^{\circ} \mathrm{C}$ qu'à celle de $80^{\circ} \mathrm{C}(0,64 \mathrm{p}$. 100/MS d'écart) et sous forme brute par rapport à celle extraite. Ces résultats indiquent que le support semble avoir un rôle de protection de l'enzyme par rapport au milieu dans lequel elle agit. De plus, ils montrent que les valeurs optimales d'activité ne se situent pas dans la fourchette située entre 60 et $80^{\circ} \mathrm{C}$ comme la firme Sigma l'indique. Cette constatation montre que l'enzyme A 1278 est moins stable thermiquement que l'enzyme A 6505 et qu'elle doit être utilisée à plus basse température que la précédente.

Les teneurs en résidus NDF, obtenues après prétraitement par l'enzyme A 1278 (sous forme non extraite), sont directement proportionnelles aux valeurs obtenues en utilisant l'enzyme A 6505 . Les coefficients de régression ne sont pas statistiquement différents de 1 ; cependant, celui relatif à l'extraction à température élevée $(1,04)$ est plus éloigné de cette valeur que celui $(0,99)$ concernant la température basse. Il est donc possible de tranformer les valeurs obtenues avec l'une de ces enzymes pour les comparer avec celles obtenues avec l'autre, et notamment, d'homogénéiser les résultats concernant les différentes matières premières, puisque, seule, l'enzyme A 1278 est actuellenent disponible sur le marché. Une telle transformation n'est pas possible avec l'enzyme Calbiochem qui engendre une relation statistiquement différente de 1 , ce qui signifierait que cette enzyme très purifiée a un spectre d'activités différent de celui des enzymes de Sigma.

En enlevant de la teneur en résidu NDF la valeur de l'amidon qu'il contient, on obtient une teneur de résidu «NDF désamidonné ». L'étude des variations de ces valeurs laisse supposer que les préparations enzymatiques possèdent des activités autres qu'amylasiques (tabl. 3). Afin de préciser cette hypothèse de travail, nous avons calculé par différence la teneur en constituants pariétaux des aliments étudiés en soustrayant d'un total de 100, les teneurs en matières azotées totales, extrait éthéré, substances pectiques, glucides alcoolosolubles et cendres déterminées dans le présent travail ou fournies par les tables (Demarquilly, Andrieu \& Sauvant, 1978 et Thivend, 1981). Cette teneur en "constituants pariétaux " estimés a été comparée aux valeurs du résidu NDF obtenu sans ou avec un prétraitement enzymatique (A 6505 ou A 1278, sans extraction et à $45^{\circ} \mathrm{C}$ ). 
TABLEAU 3

Teneurs en NDF « désamidonné».

Starchyless NDF.

\begin{tabular}{|c|c|c|c|c|c|}
\hline \multirow{4}{*}{$\begin{array}{l}\text { Aliment } \\
\text { Feed }\end{array}$} & \multirow{4}{*}{$\begin{array}{l}\text { NDF } \\
\text { Direct }\end{array}$} & \multicolumn{4}{|c|}{$\begin{array}{l}\text { NDF mesuré après traitement enzymatique } \\
\text { NDF measured after enzymatic treatment }\end{array}$} \\
\hline & & \multirow{3}{*}{ A 6505} & \multirow{2}{*}{\multicolumn{2}{|c|}{ 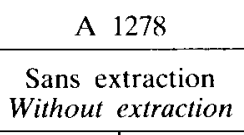 }} & \multirow{3}{*}{ Calbiochem } \\
\hline & & & & & \\
\hline & & & T 90 & T 45 & \\
\hline Maïs - Maize & 13,3 & 10,3 & 11,3 & 10,6 & 11,2 \\
\hline Blé - Wheat & 12,7 & 9,0 & 12,3 & 9,6 & 12,8 \\
\hline Orge - Barley & 20,5 & 16,4 & 17,6 & 15,9 & 18,4 \\
\hline Escourgeon - Winter barley & 20,4 & 16,6 & 16,0 & 15,6 & 19,2 \\
\hline Avoine - Oats & 28,0 & 22,9 & 23,5 & 24,4 & 22,8 \\
\hline Pois-Peas . . & 26,3 & 8,7 & 11,6 & 10,6 & 11,3 \\
\hline $\begin{array}{l}\text { Aliment composé } \ldots \ldots \\
\text { Compound feed }\end{array}$ & 19,8 & 15,7 & 16,2 & 14,8 & 19,0 \\
\hline Féverole - Field beans & 12,3 & 16,7 & 20,5 & 16,8 & 18,7 \\
\hline Son-Bran ..... & 55,2 & 48,1 & 52,3 & 47,3 & 52,6 \\
\hline $\begin{array}{l}\text { Drèches de brasserie } \\
\text { Brewers grain }\end{array}$ & 62,9 & 62,0 & 62,2 & 61,4 & 64,4 \\
\hline $\begin{array}{l}\text { Pulpes de betteraves } \\
\text { Sugar beet pulp }\end{array}$ & 49,9 & 50,2 & 49,4 & 46,7 & 48,8 \\
\hline
\end{tabular}

Tous les résultats sont exprimés en \%/MS - All results are expressed as \%/DM.

Les valeurs estimées et calculées à partir du résidu NDF n'ayant pas subi de prétraitement enzymatique sont assez proches, sauf pour les drèches de brasserie, le son et le pois. La surestimation du résidu NDF est probablement due, dans ces cas, soit à des contaminations protéiques et lipidiques, notamment dans le cas des drèches, soit à des erreurs dans le calcul de l'estimation des constituants pariétaux, ce qui ne serait pas étonnant avec des produits aussi peu courants que les drèches de brasserie ou le pois dont la composition chimique est beaucoup moins bien connue que celle des autres aliments.

Les enzymes A 1278 et A 6505 semblent avoir une action similaire. Elles possèdent probablement des activités cellulasiques et hémicellulasiques comme BAKER, Norris \& Lı (1979) l'avaient déjà observé sur du son. Les traitements utilisés ne semblent pas suffisants pour extraire la totalité de l'azote, comme le montre le cas de l'échantillon «drèches de brasserie » pour lequel le résidu NDF peut contenir jusqu'à 20 ou 30 p. 100 de matières azotées totales (Aufrere, communication personnelle).

Cependant, les comparaisons faites sont à considérer avec prudence, dans la mesure où la teneur en constituants pariétaux a été estimée et non mesurée. 
P0 Pesée de $1 \mathrm{~g}$ d'échantillon dans un creuset en verre fritte.

Weighing of $1 \mathrm{~g}$ sample in a fritted glass crucible.

Hydrolyse pendant $10^{\prime}$ dans de l'eau distillée à ébullition.

Hydrolysis for $10^{\prime}$ in boiling distilled water.

Addition d'eau distillée froide pour porter la solution à une température d'environ $45^{\circ} \mathrm{C}$. Addition of cold distilled water in order to chill the solution to $45^{\circ} \mathrm{C}$.

Addition de $250 \mathrm{mg}$ d'amylase (A 1278, Sigma) et laisser agir pendant 30'.

Addition of $250 \mathrm{mg}$ amylase (A 1278, Sigma Ltd), and let stand for 30'.

Filtration puis hydrolyse pendant 1 heure avec le détergent neutre.

Filtration and hydrolysis for 1 hour with a neutral detergent solution.

P1 Séchage du résidu pendant 8 heures à $103{ }^{\circ} \mathrm{C}$ et pesée du résidu.

Drying of residue at $103^{\circ} \mathrm{C}$ for 8 hours and weighing of residue.

Hydrolyse pendant 1 heure avec une solution de détergent acide.

Hydrolysis for 1 hour with an acid detergent solution.

P2 Séchage du résidu pendant 8 heures à $103^{\circ} \mathrm{C}$ et pesée du résidu.

Drying of residue at $103^{\circ} \mathrm{C}$ for 8 hours and weighing of residue.

Digestion par $\mathrm{H}_{2} \mathrm{SO}_{4} 72$ p. 100 pendant 3 heures.

Digestion for 3 hours in $72 \mathrm{p} .100 \mathrm{H}_{2} \mathrm{SO}_{4}$.

P3 Séchage du résidu pendant 8 heures à $103^{\circ} \mathrm{C}$ et pesée du résidu. Drying of residue at $103^{\circ} \mathrm{C}$ for 8 hours and weighing of residue.

Minéralisation pendant 3 heures à $550{ }^{\circ} \mathrm{C}$.

Ashing at $550^{\circ} \mathrm{C}$ for 3 hours.

P4 Pesée du résidu.

Weighing of residue.

$$
\mathrm{NDF}=[(\mathrm{P} 1-\mathrm{P} 4) / \mathrm{P} 0] \times 100
$$

FIG. 1

Mode d'obtention du résidu NDF

avec un appareil de dosage semi-automatique (Fibertec).

NDF residue obtention with a semi-automatic device (Fibertec).

\section{Conclusion}

De cette étude, il ressort que les enzymes amylolytiques utilisées permettent d'obtenir des résidus NDF exempts d'amidon, sauf dans le cas des légumineuses (pois, féverole). L'enzyme A 1278 est celle qui se rapproche le plus de l'enzyme A 6505, mais elle est plus sensible à la chaleur que la précédente et doit donc être utilisée à une température plus basse $\left(45^{\circ} \mathrm{C}\right)$.

Le protocole opératoire, décrit par Giger (et al., 1979), est modifié suivant le descriptif joint (fig. 1). 


\author{
Summary \\ Effect of different amylolytic pretreatments \\ on NDF content in feedstuffs
}

The initial VAN SoEST method (VAN SoEst \& WINE, 1967) designed for roughages can lead to an overestimation of cell-wall constituent contents (estimated as NDF residues), especially in the case of starch rich feeds. To overcome this deficiency RoBERTSON \& VAN SoEst (1977) suggested the use of a bacterial $\alpha$-amylase.

The aim of this study was to compare the influence of three amylolytic enzymes used at higher concentrations than those generally used, on NDF content of feedstuff samples, the extent of starch contamination of NDF residue and the possible effects (not of amylolytic origin) of these enzymes. Samples tested were very different in their starch content.

The A 1278 amylase (Sigma Ltd) used at $45^{\circ} \mathrm{C}$ allowed to extract almost all starch contained in the different samples, except from leguminosous seeds, as shown by starch content of NDF residue (Table 1). Results obtained with this enzyme were satisfactory and similar to those obtained with the A 6505 (Table 2). Measurement of starch content of NDF residue obtained with or without amylolytic treament showed that enzymes possessed cellulasic and hemicellulasic activities (Table 3 ). This study allowed to develop a method of analysis of simple or compound feeds providing a solution to filtration difficulties related to starch content (fig. 1).

Key words : Cell-wall, estimation, amylolytic pretreatment, carbohydrate contamination.

\title{
Références bibliographiques
}

BAKER D., NorRis K.H., Li B.W., 1979. Food fibre analysis: advances in methodology. In : "Dietary fibres: chemistry and nutrition" (ed. G.E. Inglett, I. FalkehaG), 67-78. Acad. Press, Inc., New York (U.S.A.), 285 p.

C.E.E., 1981. Adaptation of the Van Soest method to a routine determination of concentrate feedstuffs. Commission of European Communities Workshop on Methodology of Analysis of Feedingstuffs for Ruminants. European Van Soest ring Test. Report. (ed. J. VAN DER Meer). M.A.F.F., Slough Laboratory (Grande-Bretagne), 30 p.

Demarquilly C., Andrieu J., Sauvant D., 1978. Composition et valeur nutritive des aliments. In : Alimentation des ruminants» (ed. R. JARRIGE), 469-518. INRA publications, Versailles (France), $621 \mathrm{p}$.

Duprat F., Gallant D., Guilbot A., Mercier C., Robin J.P., 1980. L'amidon. In : "Les polymères végétaux et alimentaires non azotés" (ed. B. Monries), 176-231. Bordas, Paris, $345 \mathrm{p}$.

Giger S., 1979. Some aspects of the assessment of plant cell-wall constituents by the Van Soest method. In Focus, 5, 6-7.

Giger S., 1980. Evaluation des constituants pariétaux à partir des techniques proposées par VAN Soest. Document interne, $5 \mathrm{p}$.

Giger S., Sauvant D., Dorléans M., Morand-Fehr P., 1979. Détermination semi-automatique des constituants membranaires par la méthode de VAN SOEST. $30^{\circ}$ Réunion annuelle de la Fez, 2326 juillet 1979, Harrogate (Grande-Bretagne), 8 p.

Mascarenhas Ferreira A., Kerstens J., Gast C.H., 1983. The study of several modifications of the neutral detergent fibre procedure. Anim. Feed Sci. Technol., 9, 19-28.

Robertson J.B., VAN Soest P.J., 1977. Dietary fibre estimation in concentrate feedstuffs. In : 69th Meeting of the am. Soc. Anim. Sci. July, 1977, 2 p. 
Thivend P., 1981. Les constituants glucidiques des aliments concentrés et des sous-produits. In : "Prévision de la valeur nutritive des aliments des ruminants"(ed. C. Demarquilly), 219-236. INRA publications, Versailles (France), $580 \mathrm{p}$.

Thivend P., Mercier C., Guilbot A., 1965. Dosage de l'amidon dans les milieux complexes. Ann. Biol. Anim. Biochim. Biophys., 5, 513-526.

VAN Soest P.J., Wine R.H., 1967. Use of detergents in the analysis of fibrous feeds. V. Determination of plant cell-wall constituents. J. Assoc. off. Anal. Chem., 50, 50-55. 